\title{
Pelvic Insufficiency Fracture of Cervical Cancer After Radiotherapy: A Case Report and Literature Review
}

\author{
Wu xiaoxin ${ }^{1}$, Ran pengcheng ${ }^{2}$, Li gong ${ }^{3}$ and Gao lei*3 \\ ${ }^{1}$ Oncology graduate student of the Second affliated Hospital of Guangzhou University of Chinese Medicine, China \\ ${ }^{2}$ Nuclear medicine department, The Second affliated Hospital of Guangzhou University of Chinese Medicine, China \\ ${ }^{3}$ Radiotherapy department, The Second affliated Hospital of Guangzhou University of Chinese Medicine, China
}

*Corresponding author: Gao lei, Radiotherapy department, The Second affliated Hospital of Guangzhou University of Chinese Medicine, China

\section{ARTICLE INFO}

Received: 蔧 January 30, 2019

Published: 㹃 February 06, 2019

\section{ABSTRACT}

Citation: Wu xiaoxin, Ran pengcheng, Li g, Gao l. Pelvic Insufficiency Fracture of Cervical Cancer After Radiotherapy: A Case Report and Literature Review. Biomed J Sci \& Tech Res 14(1)-2019. BJSTR. MS.ID.002502.

\section{Introduction}

Radiotherapy is one of the main treatments for cervical carcinoma, which can effectively control the development of cancer and prolong the survival time of patients. With the prolongation of survival time of cervical carcinoma patients, the long-term complications caused by radiotherapy, including radiation proctitis, radiation cystitis and bone damage after Intensity modulated radiotherapy, have attracted increasing attention. In this paper, a case of cervical carcinoma fracture after radiotherapy was reported, and the related literature was reviewed. The purpose was to explore the criteria and influencing factors of pelvic insufficiency fracture.

\section{Case Report}

A 62-year-old patient who menopause in 2006 and had no discomfort such as vaginal bleeding or fluid after menopause. From August 13, 2016, she started with a small amount of vaginal bleeding, no vaginal discharge, without other discomforts. Cervical biopsy pathology suggested cervical squamous cell carcinoma, the stage is I B2. This patient received intravenous chemotherapy with TP regimen (paclitaxel $240 \mathrm{mg}$ + cisplatin $120 \mathrm{mg}$ D1-2) on August 19, 2016 and September 9, 2016 respectively. From October 12, 2016 to November 21, 2016, the patient received 25 times of pelvic Intensity Modulated Radiation Therapy (IMRT), GTVnd1: paraaortic lymph node; GTVnd2: Paravenous lymph nodes of inferior vena cava; GTVnd3, 4: Obturator lymph nodes both left and right, CTV: Drainage area of pelvic lymph nodes, include: from Inferior border of L1 to Drainage area of lymph nodes in inferior margin of obturator: Lymph node drainage area of para-abdominal aorta, lymph node drainage area of obturator, Lymph node drainage area of internal iliac artery, Lymph node drainage area of external iliac artery dose PGTVnd1,2,3,4: 6000cGy/25,PCTV:4500cGY/25 , Controlling rectal dose V30 less than 50\% Bilateral femoral head V30 less than 50\%,Bladder V30 less than 50\%; She received $40 \mathrm{mg}$ nedaplatin concurrent chemotherapy in 201610-18 during radiotherapy and four times of brachytherapy after radiotherapy(2016-11-15,2016-11-22,2016-11-25,2016-11-29). The patient was discharged after completing all the treatment and regularly reviewed complete abdominal MRI regularly in201702-27, 2017-06-05, 2017-09-05, 2017-12-04, 2018-3-16, 20186-22and2018-3-16. Diagnostic opinion: Cervical morphology slightly enlarged, a small amount of pelvic effusion, the signal is slightly uneven, all considering the changes after radiotherapy, no clear signs of recurrence and metastasis were found. The patient began to suffer from hip pain in August 2018 and bone metastasis was not completely ruled out by complete abdominal MRI. No abnormalities were found in the related cancer indicators. For definite diagnosis, PET-CT was performed Radioactive uptake of lumbosacral vertebrae and residual pelvic bones was sparse and decreased, which was changed after radiotherapy.

The bone density of the right sacroiliac joint was uneven, showing patchy slightly high-density shadow and patchy osteolytic low-density focus. The image showed increased radioactivity uptake 
of the focus, SUVmax: 5.16, longitudinal cable-like distribution along articular surface. Diagnostic Opinion: No clear signs of residual or recurrent tumors were found in cervix. Metabolism of lumbosacral vertebrae and residual pelvis was sparse and decreased, which was changed after radiotherapy. Local bone changes and increased metabolism of the right sacroiliac joint under the sacral articular surface and the right side of the S2 vertebral body were considered as pelvic insufficiency fracture. Exclude the possibility of bone metastasis and considered hip pain caused by insufficiency fracture.

\section{Discussion}

For patients with cervical cancer, radiotherapy can effectively reduce the recurrence of tumors and prolong the survival time of patients, regardless surgery or not. But radiation proctitis, radiation cystitis and insufficiency fracture after radiotherapy have a great impact on the quality of life of patients. In this case, the patient felt hip pain two years after radiotherapy, and bone metastasis could not be excluded by total abdominal MRI. For definite diagnosis, PET-CT showed Pelvic Failure Fracture (PIF) (Figures 1 \& 2). Insufficiency fracture is a kind of stress fracture, it occurs mostly in bone tissue with reduced bone mineral content and reduced bone elastic resistance. It often occurs under normal stress [1]. Reasons for impaired bone elastic resistance include: Osteoporosis, Radiation and Hormone Therapy, Rheumatoid Arthritis, Paget's Diseases, hyperparathyroidism, rickets, etc. [2]. PIF has no typical clinical symptoms, no obvious history of trauma, and complex pelvic structure, which often leads to missed diagnosis and misdiagnosis.

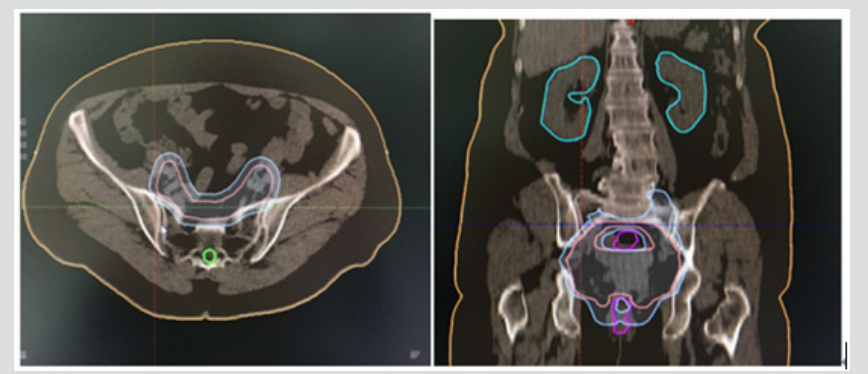

Figure 1: Radiotherapy Targe.

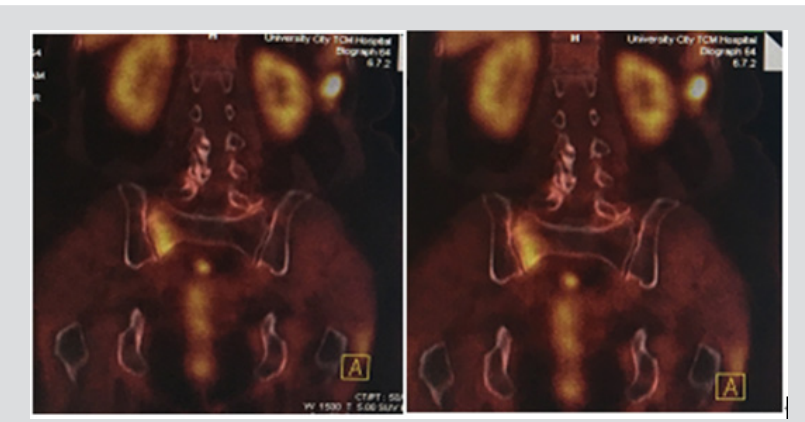

Figure 2: PET-CT.

Insufficiency fracture should be differentiated from other bone diseases, bone metastases are mostly sporadic, with uncertain size, shape and location.
Insufficiency fracture is the mainly located in the load-bearing area, the distribution is bilateral and symmetrical, in which "serpentine" can be seen Fracture line, the symptoms of fracture are mild and have its specific site of onset. Other benign and malignant bone tumors, such as osteosarcoma and osteoid bone tumors, giant cell tumors of bone and chordoma are less symmetrical. It can be differentiated by clinical symptoms and imaging examination. Clinically, it is not uncommon to have insufficiency fracture after radiotherapy, however, the current understanding of post-radiotherapy insufficiency fracture is insufficient, often lead to misdiagnosed as bone metastasis or infection. Several studies investigating the incidence of and risk factors for PIF have shown wide incidence rates ranging from $1.7 \%$ to $89 \%$ in patients undergoing treatment of gynecologic, rectal, or prostate cancer. PIF is closely related to elderly women, menopausal status, glucocorticoid use, osteoporosis and so on. More and more studies show that radiotherapy can also lead to PIF. Most PIF occur in the sacrum, sacroiliac joint and iliac bone and some occur in other parts [3-5].

Francis G. Celii report an 82-year-old woman with a remote history of right thigh basal cell carcinoma treated with resection and radiation therapy 18 years prior, presented with severe right thigh pain and inability to bear weight as she had suffered a femur fracture. Evidence pointed to radiation-induced osteoporosis as the mechanism for her insufficiency fracture [6]. For patients with cervical cancer, Sacral and sacroiliac joints are the main sites of failure fracture after radiotherapy. In recent years, with the development of radiotherapy technology, Intensity Modulated Radiation Therapy (IMRT) has been widely used in cervical cancer radiotherapy and the incidence of insufficiency fracture after radiotherapy also increased gradually. What is the mechanism of fracture failure after radiotherapy? Different people have different opinions. Shih believed that there was no significant difference in the incidence of pelvic insufficiency fracture between conventional pelvic radiotherapy and intensity-modulated radiotherapy in adjuvant radiotherapy after cervical cancer surgery [7]. Oh, considered that the insufficiency fracture of sacrum and sacroiliac joint failure might be related to the dose of radiotherapy [8].

Anne Ramlov MD considered that PIF is common after treatment of LACC and is mainly seen in patients aged $>50$ years and not related to lymph node boosts but rather to dose and volume associated with irradiation of the elective pelvic target. Reducing the prescribed elective dose from 50 to 45 Gy may reduce the risk of PIF considerably [9]. Many studies have shown that osteoporosis and postmenopausal status are important causes of insufficiency fracture, and osteoporosis is the most fundamental cause. Radiotherapy is an important inducing factor. On the basis of osteoporosis, it can aggravate the occurrence of insufficiency fracture. Blom lie reviewed 121 cases of sacral failure fracture reported in the literature, $60 \%$ of which were caused by local radiotherapy, but $95 \%$ of them occurred in elderly women after amenorrhea [10]. Mehmood 
Q consider that chemotherapeutic drugs can cause bone loss and reduced bone mineral density while treating gynecological tumors which increases the risk of fracture [11].

\section{Final Considerations}

Summarize the relevant literature, pelvic insufficiency fracture after radiotherapy for cervical cancer is not uncommon. Osteoporosis and postmenopausal status are important risk factors. There is no effective treatment for pelvic insufficiency fracture at present but by strengthening the understanding of pelvic insufficiency fracture, bone metastasis can be excluded, and inappropriate treatment can be avoided. Follow-up review is an important mean to diagnosis and differential diagnosis pelvic insufficiency fracture. Insufficiency fracture may be healed gradually, and bone destruction and soft tissue mass appeared gradually. In addition, puncture biopsy should be avoided as far as possible in the diagnosis of osteosarcoma, because less biopsy materials are available, and local immature osteocytes are easily mistaken for osteosarcoma pathologically. Biopsy can also cause local secondary infection. By study the relationship between radiotherapy dose and other physiological factors, we can reduce the incidence of pelvic failure fracture after radiotherapy.

\section{References}

1. Yalçın S, Ekiz T, Erden E, Öztürk GT, Hatipoğlu C, et al. (2015) Sacral insufficiency fracture: an insidious cause for low back pain. Pain Physician 18(5): 925-926.

2. Restan C, Hojreh A (2009) Imaging of insufficiency fractures. Eur J Radiol 71(3): 398-405.

\section{ISSN: 2574-1241}

DOI: 10.26717.BJSTR.2019.14.002502

Gao lei.Biomed J Sci \& Tech Res

This work is licensed under Creative Commons Attribution 4.0 License

Submission Link: https://biomedres.us/submit-manuscript.php
3. Higham CE, Faithfull S (2015) Bone health and pelvic radiotherapy. Clin Oncol 27(11): 668-678.

4. Schmeler KM, Jhingran A, Iyer RB, Sun CC, Eifel PJ, et al. (2010) Pelvic fractures after radiotherapy for cervical cancer: Implications for survivors. Cancer 116(3): 625-630.

5. Park SH, Kim JC, Lee JE, IK Park (2011) Pelvic insufficiency fracture after radiotherapy in patients with cervical cancer in the era of PET/CT. Radiation Oncol J 29(4): 269-276.

6. Francis G, Celii, BS, Nicholas M (2019) Radiation-induced insufficiency fracture of the femur 18 years after radiation therapy. Radiology Case Rep 14(2): 179-183.

7. Shih KK, Folkert MR, Kollmeier MA, Abu Rustum NR, Sonoda Y, et al. (2013) Pelvic insufficiency fractures in Patients with cervical and endometrial cancer treated with postoperative pelvic radiation. Gynecol Oncol 128(3): 540-543.

8. Oh D, Huh SJ, Nam H, Park W, Han Y, et al. (2008) Pelvic insufficiency fracture after pelvic radiotherapy for cervical cancer : Analysis of risk factors. Int J Radiat Oncol BiolPhys 70(4): 1183-1188.

9. Ramlov A, Pedersen EM, Rohl L, Worm E, Fokdal L, et al. (2017) Risk Factors for pelvic insufficiency fractures in locally advanced cervical cancer following intensity modulated radiation therapy. Int J Radiat Oncol Biol Phys 97(5): 1032-1039.

10. Blomlie V, Rofstad EK, Talle K, Sundfør K, Winderen M, et al. (1996) Incidence of radiation-induced insufficiency fractures of the female pelvis: Evaluation with MR imaging. AJR Am J Roentgenol 167(5): 12051210.

11. Mehmood Q Beardwood M, Swindell R, Greenhalgh S, Wareham T, et al. (2014) Insufficiency fractures in patients treated with pelvic radiotherapy and chemotherapy for uterine and cervical cancer. Eur J Cancer Care (Engl) 23(1): 43-50.

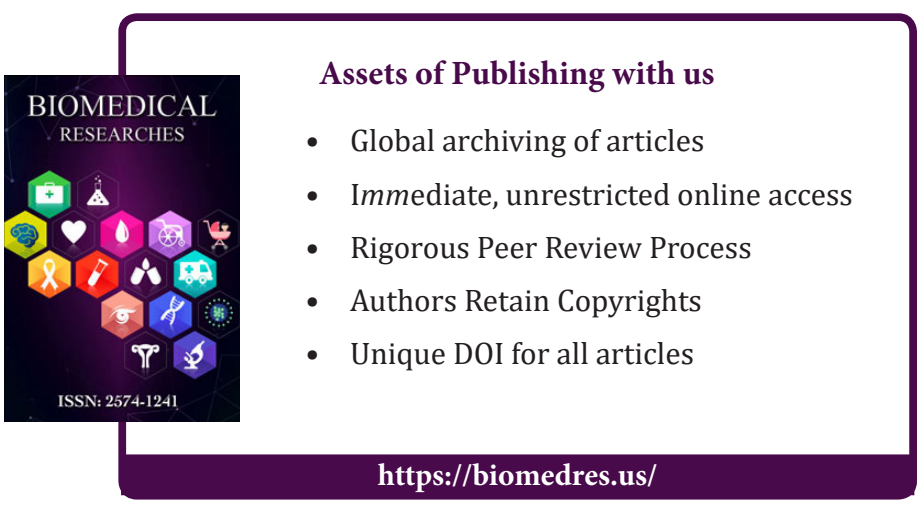

